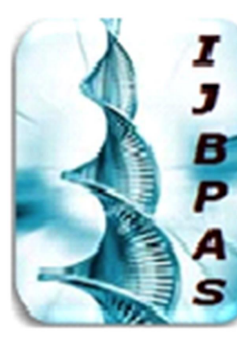

International Journal of Biology, Pharmacy and Allied Sciences (IJBPAS) 'A Bridge Betueen caboratory med QRendo'

WwW.ibpas.com

\title{
PNEUMOTHORAX IN COVID 19 POSITIVE PATIENT
}

NIGANIA RR ${ }^{1}$, NERLEKAR HV ${ }^{2}$ AND AJAGEKAR PD ${ }^{3}$

1: Junior Resident, Department of Surgery, Krishna Institute of Medical Sciences Deemed

To Be University, Karad, Maharashtra (India)

2: Associate Professor, Department of Surgery, Krishna Institute of Medical Sciences Deemed To Be University, Karad, Maharashtra (India)

3: Senior Resident, Department Of Surgery, Krishna Institute of Medical Sciences Deemed To Be University, Karad, Maharashtra (India)

*Corresponding Author: Dr. Nigania Rashika Rajesh: E Mail: rashika1995.12@gmail.com Received $15^{\text {th }}$ July 2021; Revised $18^{\text {th }}$ Aug. 2021; Accepted $29^{\text {th }}$ Oct. 2021; Available online $15^{\text {th }}$ Feb. 2022

\section{https://doi.org/10.31032/IJBPAS/2022/11.2.1027}

\section{ABSTRACT}

\section{OBJECTIVE}

- To discover the prevalence and consequences of pneumothorax in patients admitted to our group who are suspected of having COVID-19 pneumonia.

\section{STUDY DESIGN}

- Retrospective study.

\section{SETTING}

- Krishna Hospital and Research Center, Karad.

\section{SUBJECTS AND METHODS}

- A study was performed including the patients admitted to KIMS hospital for COVID19.

- The study then identified the patients admitted who were COVID-19 positive and were found to develop spontaneous pneumothorax to calculate the incidence of this event.

- The signs and symptoms of such patients were then studied.

- Finally, the outcomes of the clinical trials were carefully studied. 


\section{RESULT}

- The initial sample size included 1000 patients suspected to have COVID-19 pneumonia.

- Out of these 1000 suspected patients, 604 had positive RTPCR reports suggesting the presence of the novel Corona virus.

- Out of the 604 COVID-19 positive patients, the patients that developed spontaneous pneumothorax were 5 .

- The initial CT SCAN showed ground glass opacities and consolidations in both lungs. This was found to be more prominent in the posterior and peripheral lung regions.

- Out of the 5 cases with spontaneous pneumothorax, 3 were related to mechanical ventilation.

- All the patients include in the study required ICDT insertion.

- Pneumothorax seemed to be indirectly correlated with the mortality rate of $66.2 \%$.

\section{CONCLUSION}

- The conclusion of the study is that a rare complication amongst patients suffering from COVID-19 pneumonia is the spontaneous pneumothorax.

- The spontaneous pneumothorax may occur in the presence or absence of mechanical ventilation.

\section{INTRODUCTION}

- Spontaneous pneumothorax suggests presence of air in the pleural space. This space should not be caused by iatrogenic factors such as trauma or other obvious precipitating factor.

- Such primary spontaneous pneumothorax is identified as the ones that occur when there is no existing lung conditions.

- While the secondary spontaneous pneumothorax generally occurs due to complications in existing lung disease $[\mathbf{1}, \mathbf{2}]$.

- The studies till date have not mentioned or identified pneumothorax as a condition which can cause complications in patient suffering from covid 19 disease and the number of new cases of pneumothorax is yet unknown $[\mathbf{3}, \mathbf{4}$, $5,6]$.

- Yang et al. study 92 deceased COVID-19 patients. They find that $1.1 \%$ of their sample developed 
pneumothorax. They also find that these patients died 5 days after appearance of thed first symptoms [9].

- Studies have shown a link between Pneumothorax and poor prognosis in patients infected with the acute Middle East respiratory syndrome coronavirus (MERS-CoV) [10].

- The structural changes happening in the lung parenchyma is said to be a cause for the development of spontaneous pneumothorax in patients contracting the COVID-19 virus.

- These changes can be identified as fibrotic and cystic changes. These changes further lead to alveolar tears.

- Another reason for the development of spontaneous pneumothorax is the growth in intrathoracic pressure due to extended coughing and mechanical air flow $[3,5,6,10$, 11].

- Therefore, the aim of this study is to discover the prevalence and consequences of pneumothorax in patients that have been tested COVID-19 positive.

\section{MATERIALS AND METHODS}

- A study was performed including the patients admitted to KIMS hospital for COVID-19.

- The study then identified the patients admitted who were COVID-19 positive and were found to develop spontaneous pneumothorax to calculate the incidence of this event.

- We identified 604 patients who were tested positive for the novel Corona virus based on the RTPCR testing.

- As a part of the study, all patients suffering from COVID-19 had to undergo a CT SCAN of the chest along with chest x-ray after admission to our institute.

- Clinical documentation and chest radiographic imaging were used to determine the presence of pneumothorax.

- Any patient showing symptoms of a pneumothorax at any time during their stay in the hospital were studied carefully.

- Laboratory investigations like

- C-reactive Proteins (crp)

- WBC

- LDH

- Serum Ferritin

- IL-6 
- D-dimer

- SF ratio was used to evaluate the respiratory function.

- $\quad$ SF ratio $=$ oxygen saturation as measured by pulse oximetry $\left(\mathrm{SpO}_{2}\right)$

fraction of inspired oxygen $\left(\mathrm{FiO}_{2}\right)$

- We then calculated and studied the occurrence of spontaneous pneumothorax in patients suffering from COVID-19.

- The protocol for this study was approved by the Temple University Hospital Review Board.

\section{INCLUSION CRITERIA}

Following are the inclusion criteria for this study:

- Patients tested positive for COVID 19 by RTPCR test.

- Patients admitted to Krishna Hospital and Medical Research Center, Karad.

\section{EXCLUSION CRITERIA}

- Patients who are unwilling to participate.

- Patients with COVID-19 negative status.

\section{RESULTS}

- The initial sample size included 1000 patients suspected to have COVID-19 pneumonia.

- Out of these 1000 suspected patients, 604 had positive RTPCR reports suggesting the presence of the novel Corona virus.
- Out of the 604 COVID-19 positive patients, the patients that developed spontaneous pneumothorax were 5 .

- The initial CT SCAN showed ground glass opacities and consolidations in both lungs. This was found to be more prominent in the posterior and peripheral lung regions.

- Out of the 5 cases with spontaneous pneumothorax, 3 were related to mechanical ventilation.

- All the patients include in the study required ICDT insertion.

- Pneumothorax seemed to be indirectly correlated with the mortality rate of $66.2 \%$.

\section{REVIEW OF CASES}

\section{Case1:}

- Age - 50 years

- Gender - male

- Morbidly obese.

- Having CAD and heart failure.

- Came with chief complains of cough, loss of sensation of smell, fever and breathlessness.

- SF ratio 215.

- Non-smoker and no pre-existing lung disorder.

- The patient needed oxygen via nasal cannula. 
- Changes were observed in base and middle zones of both lungs using CXR.

- HRCT showed ground glass opacities like appearance and consolidations in $\mathrm{B} / \mathrm{L}$ lungs mostly in the periphery of the lungs.

- Blood investigations showed decreased WBC counts and rise in markers of inflammation.

- Antibiotics, corticosteroids, convalescent plasma and inj. Tocilizumab were administered to the patient.

- 6 days later, the patient's saturation started dropping again. The patient was then placed on a high-flow nasal cannula (HFNC).

- The possibility of Pulmonary embolism was ruled out using a CT angiogram

- The CT angiogram showed a large pneumothorax in the left lung.

- Intercostal drainage tube was inserted in the $5^{\text {th }}$ intercostal space.

- Patient's condition started to improve and the above condition started to started to get better within 14 hours.

- This patient's hospital course was further complicated by worsening hypoxia, patient was put on invasive mechanical ventilation.
- However, later the patient's condition worsened owing to multiorgan failure. Thus the patient expired.

\section{Case 2}

- Age - 60 years

- Gender-male

- Obese

- Came with chief complains of easy tiredness, raised temperature, cough and breathlessness since 12 days.

- Patient was kept on HFNC.

- The patient's SF ratio was 150 .

- Blood investigations showed decreased wbc count and rise in markers of inflammation.

- Multiple opacities were seen on both the lungs on chest xray.

- Ground glass opacity and consolidation was seen on HRCT.

- Antibiotics and corticosteroids given

- In addition, the affected person later obtained convalescent plasma and inj. Tocilizumab.

- After 2 days patient was put on mechanical ventilator.

- Ten days after intubation, tracheostomy was done. 
- Patients saturation slowly started falling and patient became unstable

- USG of the chest was done.

- It showed tension pneumothorax

- $\mathrm{c}$

- The patient's condition started to improve over time after placing the intercostal drainage tube.

- One week later patient was slowly weaned off the mechanical ventilator.

- Gradually ICDT was removed.

Case 3

- Age - 82 years

- Gender- female

- Prior history of high blood pressure and a stroke with progressive fatigue, lack of urge for food and diarrhoea was admitted.

- Blood investigations showed decreased WBC counts and rise in markers of inflammation.

- HRCT showed ground glass opacities, haziness in posterior and peripheral parts of both the lungs.

- On admission she was started on 4 litres $\mathrm{O}_{2}$ using prongs.

- The patient's SF ratio was 210.

- Various antibiotics, steroids were given to the patient.
- Later injection Remdesivir was also started for the above patient.

- Patients saturation slowly started falling and patient became unstable and condition worsened

- Patient was started on HFNC.

- The x-ray findings of the patient showed consolidation in both the lungs which was in increasing trend.

- After few days a chest X-ray was repeated which showed pneumothorax.

- Intercostal drainage tube was inserted in the $5^{\text {th }}$ intercostal space.

- Serial x-rays were done everyday which showed improvement in patient's lung condition.

- After 4 days intercostal drainage tube was removed.

- Post removal of icdt, after few days again the patient's saturation started to fall and the chest $\mathrm{x}$-ray indicated a new pneumothorax.

- However, later the patient's condition worsened further.

- The patient died due to complications.

\section{Case 4}

- Age - 42 years

- Gender - female 
- Came with chief complains of shortness of breath and cough.

- She had fever on admission and was not maintaining saturation

- $\mathrm{SpO}_{2}$ of the patient was $82 \%$

- SF ratio 133.

- Blood investigations showed Lymphopenia and rise in markers of inflammation.

- HRCT showed ground glass opacities and consolidative changes were seen in both the lungs

- Later, patient became hypoxic and need for oxygen started to rise.

- Patient was intubated.

- The chest x-ray after intubation showed intubation in the bronchus

- Left lung of the patient showed atelectatic changes.

- The endotracheal tube was pulled out to a point and fixed.

- Later on a chest X-ray was repeated to confirm the position of the tube.

- This chest $\mathrm{x}$-ray showed pneumothorax.

- Intercostal drainage tube was inserted in the $5^{\text {th }}$ intercostal space.

- Corticosteroids, antibiotics, convalescent plasma and inj. Tocilizumab was given.
- Intercostal drainage tube was removed after the patient showed recovery.

- Slowly extubatiion was planned for this patient and then the oxygen requirement reduced and finally patient was discharged.

Case 5

- Age -47 years

- Gender - female

- Obese

- Patient suffered from DM, meningioma, hypothyroid.

- The patient presented with chief complains of cough, raised temperature and easy fatiguability.

- On admission she had raised temperature, tachypnoea and hypoxia requiring oxygen through high flow nasal cannula.

- $\mathrm{SF}$ ratio of the patient was 94 .

- Blood investigations showed decreased wbc count and rise in markers of inflammation.

- Pulmonary embolism, ground glass opacity and consolidation like changes were seen in both lungs.

- Patient was given antibiotics, steroids 
- Continuous i.v. infusion of heparin was started.

- Five days afterwards, the patient became more hypoxic with increased work of breathing requiring intubation and mechanical ventilation.

- Her CXR revealed bilateral pneumothorax, (right $>$ left).

- Intercostal drainage tube was inserted in the $5^{\text {th }}$ intercostal space on right side.

- Over time the pneumothorax of the above patient started to decrease.

- Her stay in the hospital was further complicated as the patient developed kidney failure and pneumonia due to prolong use of ventilator.

- Patient expired as a result of cardio-pulmonary arrest.

\section{DISCUSSION}

- Other comorbidities involving the lung play an important role in developing pneumothorax

- The comorbidities include emphysema, COPD, Koch's, fibrosis, carcinoma of lung etc $[1,2,12]$.

- Patient positive for COVID-19 infection can at further stages develop acute respiratory distress syndrome (ARDS).

- Radiological imaging showed : ground glass opacity

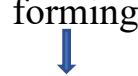

\section{Consolidation}

Fibrotic changes (later stage) $[\mathbf{8}, \quad$ 13] Various factors that worsen SARS infection is - diffuse alveolar injury Severe lung injury [14].

Increase risk for developing pneumothorax.

- Mechanical ventilation is predominant the major risk factor for developing pneumothorax in patients having COVID-19 disease.

- Aiodfi and co-workers mentioned in their study that persistent pneumothorax can be developed even in intubated patients in COVID-19 positive cases [4].

- However, Wang et al. saw that patient who was not intubated also developed spontaneous pneumothorax, emphysema and pneumomediastinum [10].

- Number of cases suffering from pneumothorax in intubated patients were quite high. 
- It is higher in intubated patients suffering from other lung comorbidities.

- The severity of pneumothorax is directly proportional to acute respiratory distress syndrome (duration and severity of the condition), trauma caused to the lung because of the ventilator.

- In Acute respiratory distress syndrome the lung shows diseased alveoli and normal alveoli.

- Interstitial edema

$\checkmark$ Causes

Consolidation in dependent parts of lung

\section{Leading to}

Decrease in lung compliance

- During lung recruitment manoeuvres while managing acute respiratory distress syndrome, overdistention of non-dependent lung regions with rather higher compliance and less airway resistance occurs.

- While treating ARDS

There is distension of the normal lung areas which has less resistance and high compliance.
There is imbalance in the distribution of volume and pressure from ventilator

\section{Leading to}

Alveoli rupture

- Out of the 5 cases with spontaneous pneumothorax, 3 were related to mechanical ventilation.

- The intubated patients had lung compliance on the higher side.

- The lung can be protected from developing pneumothorax by managing the lung volume and pressure.

- Both the volume and pressure should be kept low.

- This low pressure and volume reduces the trauma (barotrauma and volutrauma) caused to the lungs.

- Intubation showed be the last resort in treating patient and instead of that high flow oxygen should be used to minimize the complications of intubation

- Coughing is also another factor (which is a very common symptom in covid 19 patients) which can trigger complications.

- Four out of five sufferers suggested cough as a principal symptom of their presentation. 
- During cough, Because of sudden increase and decrease in length of the pulmonary vessels and bronchi there might be leaking of air from the alveoli.

- This further causes shift of train of bubbles in vascular sheaths [21].

- Radiological scans can be used as a predictive tool in patients who might develop pneumothorax.

- Radiological imaging showed atelectasis

- consolidations

- ground glass opacities [13, 23]

- These changes are also seen in similar studies done for the above condition.

- In KIMS, karad all the patients showing positive swab for COVID 19 disease was made to do HRCT, in addition to a chest $\mathrm{x}$-ray on a daily basis to guide medical decisions and medical management.

- Various laboratory investigations were also done.

- The findings were mostly as below in patients developing pneumothorax along with COVID 19 disease:
TLC count - showed decrease in

WBC.

CRP - increased.

LDH - increased.

Serum ferritin - increased.

D-dimer - increased.

IL-6 - increased.

- Cytokine storm

Cause hyperinflammatory form of ARDS

Increase in mortality $[\mathbf{2 4 , ~ 2 5 , ~ 2 6 ] . ~}$

- The lung tissue of patients who died of covid 19 pneumonia and also had acute respiratory distress syndrome showed angiopathy in the small vessels of lung and thrombus also. This factor can also be a major factor in causing lung damage; but more studies have to be done to confirm this factor as a cause [27].

- In KIMS, COVID-19 positive patients have been put on inj. Clexane or heparin to prevent various angiopathy or thrombus formation.

- The dose, duration and type of anticoagulant was decided on the level of D-dimer. 
- Damage to the pleural cavity because of damage to anterior or posterior tracheal wall is thought to be the cause behind pneumothorax [28, 29].

- No damage to the tracheal was seen in our study group.

- So the cause of pneumothorax could be inflammation to the lung parenchyma, positive pressure ventilation in intubate patients and impaction of mucus.

- Mucus impaction and inflammation of alveoli can cause over-distension of the alveoli can increase the risk of developing pneumothorax in covid 19 positive patients.

- Although that is a rare problem at some stage in tracheostomy placement, proceduralists should be aware that patients suffering from COVID 19 disease is at higher risk for developing pneumothorax and a special care ought to be taken when dealing with airways of those patients.

\section{CONCLUSION}

- The conclusion of the study is that a rare complication amongst patients suffering from COVID-19 pneumonia is the spontaneous pneumothorax.

- The spontaneous pneumothorax may occur in the presence or absence of mechanical ventilation.

\section{ACKNOWLEDGEMENT}

- I am thankful to Mrs. Rupali Salunkhe for secretariat help, Institute of Medical Sciences, Deemed to be University, Karad, Maharashtra (India).

\section{REFERENCES}

[1] MacDuff A, Arnold A, Harvey J, Management of spontaneous pneumothorax: British Thoracic Society pleural disease guideline 2010, Thorax, 65(2), 2010, ii18-31.

[2] Baumann $\mathrm{MH}$, Management of spontaneous pneumothorax, Clinics in chest medicine, 27(2), 2006, 369-81.

[3] Zhou C, Gao C, Xie Y, Xu M, COVID-19 with spontaneous pneumomediastinum, The Lancet Infectious Diseases, 20(4), 2020, 510 .

[4] Aiolfi A, Biraghi T, Montisci A, Bonitta G, Micheletto G, Donatelli F, et al., Management of persistent pneumothorax with thoracoscopy and bleb resection in COVID-19 patients, The Annals of thoracic surgery, 110(5), 2020, e413-5.

[5] Wang J, Su X, Zhang T, Zheng C, Spontaneous pneumo-mediastinum: a probable unusual complication of coronavirus disease 2019 (COVID-19) 
pneumonia, Korean Journal of Radiology, 21(5), 2020, 627.

[6] Sun R, Liu H, Wang X, Mediastinal emphysema, giant bulla, and pneumothorax developed during the course of COVID-19 pneumonia, Korean Journal of Radiology, 21(5), 2020, 541.

[7] Chen N, Zhou M, Dong X, Qu J, Gong F, Han Y, et al., Epidemiological and clinical characteristics of 99 cases of 2019 novel coronavirus pneumonia in Wuhan, China: a descriptive study, The lancet, 395(10223), 2020, 507-13.

[8] Yang F, Shi S, Zhu J, Shi J, Dai K, Chen $\mathrm{X}$, Analysis of 92 deceased patients with COVID-19, Journal of medical virology, 92(11), 2020, 2511-5.

[9] Das KM, Lee EY, Jawder SE, Enani MA, Singh R, Skakni L, et al., Acute Middle East respiratory syndrome coronavirus: temporal lung changes observed on the chest radiographs of 55 patients, American Journal of Roentgenology, 205(3), 2015, W267-S274.

[10] Wang W, Gao R, Zheng Y, Jiang L, COVID-19 with spontaneous pneumothorax, pneumomediastinum and subcutaneous emphysema, Journal of travel medicine, 27(5), 2020, taaa062.

[11] Liu K, Zeng Y, Xie P, Ye X, Xu G, Liu J, et al., COVID-19 with cystic features on computed tomography: a case report. Medicine, 99(18), 2020.

[12] Noppen M, Spontaneous pneumothorax: epidemiology, pathophysiology and cause,
European Respiratory Review, 19(117), 2010, 217-9.

[13] Hosseiny M, Kooraki S, Gholamrezanezhad A, Reddy S, Myers L, Radiology perspective of coronavirus disease 2019 (COVID-19): lessons from severe acute respiratory syndrome and Middle East respiratory syndrome, American Journal of Roentgenology, 214(5), 2020, 1078-82.

[14] Sihoe AD, Wong RH, Lee AT, Lau LS, Leung NY, Law KI, et al., Severe acute respiratory syndrome complicated by spontaneous pneumothorax, Chest, 125(6), 2004, 2345-51.

[15] Albelda SM, Gefter WB, Kelley MA, Epstein DM, Miller WT, Ventilatorinduced subpleural air cysts: clinical, radiographic, and pathologic significance. American Review of Respiratory Disease, 127(3), 1983, 360-5.

[16] Gammon RB, Shin MS, Buchalter SE, Pulmonary Barotrauma in Mechanical Ventilation, Chest, 104(3), 1993, 987.

[17] Woodside KJ, vanSonnenberg E, Chon KS, Loran DB, Tocino IM, Zwischenberger JB, Pneumothorax in patients with acute respiratory distress syndrome: pathophysiology, detection, and treatment, Journal of intensive care medicine, 18(1), 2003, 9-20.

[18] Gattinoni L, Chiumello D, Caironi P, Busana M, Romitti F, Brazzi L, et al., COVID-19 pneumonia: different respiratory treatments for different phenotypes?, 
[19] Guan WJ, Ni ZY, Hu Y, Liang WH, Ou CQ, He JX, et al., Clinical characteristics of coronavirus disease 2019 in China, New England journal of medicine, 382(18), 2020, 1708-20.

[20] Tian S, Hu N, Lou J, Chen K, Kang X, Xiang Z, et al., Characteristics of COVID19 infection in Beijing. Journal of infection, 80(4), 2020, 401-6.

[21] Macklin CC, Transport of air along sheaths of pulmonic blood vessels from alveoli to mediastinum: clinical implications, Archives of Internal Medicine, 64(5), 1939, 913-26.

[22] McClellan MD, Miller SB, Parsons PE, Cohn DL, Pneumothorax with Pneumocystis carinii pneumonia in AIDS: incidence and clinical characteristics, Chest, 100(5), 1991, 1224-8.

[23] Bernheim A, Mei X, Huang M, Yang Y, Fayad ZA, Zhang $\mathrm{N}$, et al., Chest CT findings in coronavirus disease-19 (COVID-19): relationship to duration of infection, Radiology, 2020, 200463.

[24] QinC Z, Dysregulation of immune response in patients with COVID-19 in Wuhan, China, Clin Infect Dis, 2020.

[25] Giamarellos-Bourboulis EJ, Netea MG, Rovina N, Akinosoglou K, Antoniadou A, Antonakos $\mathrm{N}$, et al., Complex immune dysregulation in COVID-19 patients with severe respiratory failure, Cell host \& microbe, 27(6), 2020, 992-1000.

[26] Ye Q, Wang B, Mao J, The pathogenesis and treatment of the Cytokine Storm'in
COVID-19, Journal of infection, 80(6), 2020, 607-13.

[27] Ackermann M, Verleden SE, Kuehnel M, Haverich A, Welte $\mathrm{T}$, Laenger $\mathrm{F}$, et al., Pulmonary vascular endothelialitis, thrombosis, and angiogenesis in Covid-19, New England Journal of Medicine, 383(2), 2020, 120-8.

[28] Panajaroen P, Tangjaturonrasme N, Pneumothorax after tracheostomy: a prospective study, Otolaryngol Pol, 69(3), 2015, 26-30.

[29] Li YW, Chandan GS, Bilateral tension pneumothoraces following percutaneous tracheostomy, Journal of the Intensive Care Society, 10(4), 2009, 295-6. 